\title{
Tunable monoenergetic electron beams from independently controllable laser-wakefield acceleration and injection
}

\author{
G. Golovin, ${ }^{1}$ S. Chen, ${ }^{1}$ N. Powers, ${ }^{1}$ C. Liu, ${ }^{1}$ S. Banerjee, ${ }^{1}$ J. Zhang, ${ }^{1}$ M. Zeng, ${ }^{2}$ \\ Z. Sheng, ${ }^{2}$ and D. Umstadter ${ }^{1, *}$ \\ ${ }^{1}$ Diocles Extreme Light Laboratory, University of Nebraska-Lincoln, Lincoln, Nebraska 68508, USA \\ ${ }^{2}$ Department of Physics, Shanghai Jiao Tong University, Shanghai 200240, China
}

(Received 7 February 2014; published 5 January 2015)

\begin{abstract}
We report the results of experiments on laser-wakefield acceleration in a novel two-stage gas target with independently adjustable density and atomic-composition profiles. We were able to tailor these profiles in a way that led to the separation of the processes of electron injection and acceleration and permitted independent control of both. This resulted in the generation of stable, quasimonoenergetic electron beams with central energy tunable in 50-300 MeV range. For the first time, we are able to independently control the beam charge and energy spread over the entire tunability range.
\end{abstract}

Over the past several decades, the physics of laserwakefield acceleration (LWFA) has been the subject of intense investigation [1-5]. The impetus for this interest has been LWFA's potentially transformative features, several of which have been demonstrated experimentally, including an orders-of-magnitude higher acceleration gradient (compared to conventional radio-frequency linear accelerators), monoenergicity, and femtosecond pulse duration. Among other applications, LWFA has been integral to the development of compact $\mathrm{x}$-ray sources based on various mechanisms, such as betatron radiation [6,7], conventional undulator-based synchrotron radiation [8,9], and inverse Compton scattering [10-12]. Independent control of the electron beam parameters is one of the most significant advances still needed in order for these applications to become practical.

There has been recent progress towards achieving this goal using the following approaches: (i) optical injection, involving two independent laser pulses, one to drive the wake and the other to inject electrons [13-17]; (ii) plasmaprofile tailoring, either by means of a machining laser pulse [18-20] or by the introduction of obstacles into the gas flow [21-23]; or (iii) the use of distinct media for injection and acceleration [24-28]. While these methods have resulted in tunable quasimonoenergetic electron beams, none of them were able to control the energy spread and charge as the beam was tuned in energy. This primarily arose from the lack of independent control of both the injection and acceleration processes.

\section{"donald.umstadter@unl.edu}

Published by the American Physical Society under the terms of the Creative Commons Attribution 3.0 License. Further distribution of this work must maintain attribution to the author $(s)$ and the published article's title, journal citation, and DOI.
We report here an experimental study showing that the injection and acceleration processes can be independently controlled by the use of a single laser pulse focused onto a composite gas target, created by two overlapped gas jets, with independently adjustable gradient profiles of gas density and atomic composition. We were able to create profiles with three distinct regions. In the first region, the plasma wave grew and an acceleration bucket was formed. In the second region, ionization-assisted injection was localized. Finally, in the third region, acceleration and electron-bunch phase-space rotation occurred. As a result, we were able to tune $e$-beam energy while having control over both the electron charge and energy spread. The stable, quasimonoenergetic, and tunable $e$ beams proved to be critical for generating quasimonoenergetic and tunable $\mathrm{x}$ rays through inverse Compton scattering [12].

The experiments were performed with the DiOCLES laser system [29] (Ti:sapphire, 805-nm central wavelength, $10-\mathrm{Hz}$ repetition rate), delivering $34-\mathrm{fs}$ pulses with $1.7 \mathrm{~J}$ (47 TW peak power) of laser energy on target. A closedloop technique for temporal phase correction allowed for Fourier-transform-limited pulse duration [30]. An $f / 14$ off-axis paraboloid (1-m focal length) focused the laser pulses to a $20-\mu \mathrm{m}$ diameter (FWHM) focal spot with $70 \%$ of the energy contained within the $1 / e^{2}$ contour. A deformable mirror corrected the optical aberrations of the laser wave front resulting in a Strehl ratio of 0.9.

Gas-target profiles were formed by merging two supersonic jets. We used a 0.5 -mm-long first nozzle and 0.5 - or 2-mm-long second nozzle (both slits), separated by a 0.5-mm gap [see the inset in Fig. 1(a)]. The laser beam focal point was located between the nozzles, at a height of $2 \mathrm{~mm}$ above the nozzle orifices. This double-nozzle configuration allowed us to independently tailor the profiles of both the gas density and atomic composition. Gas density profiles were measured off-line (see Fig. 1(a) and 
(a)

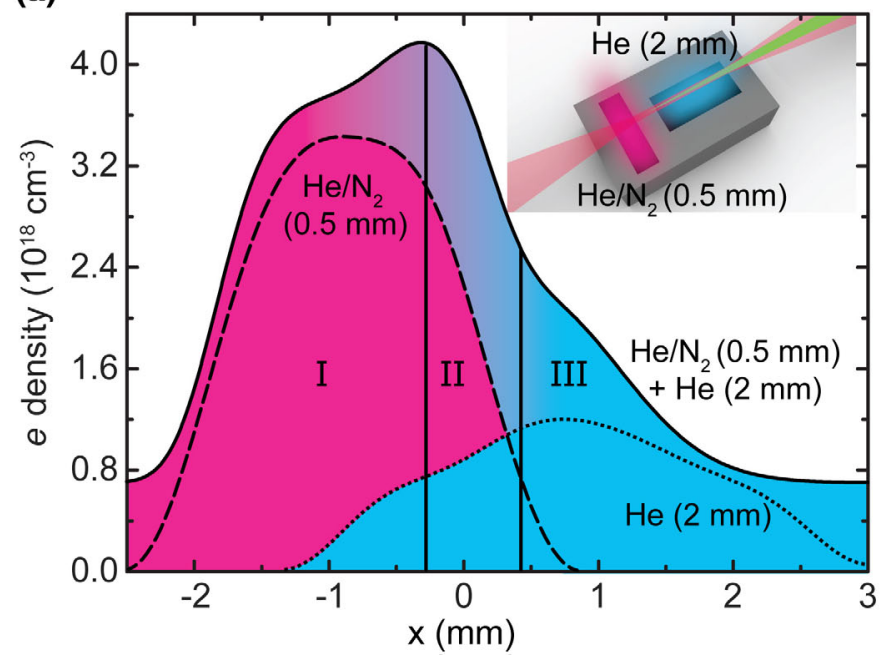

(b)

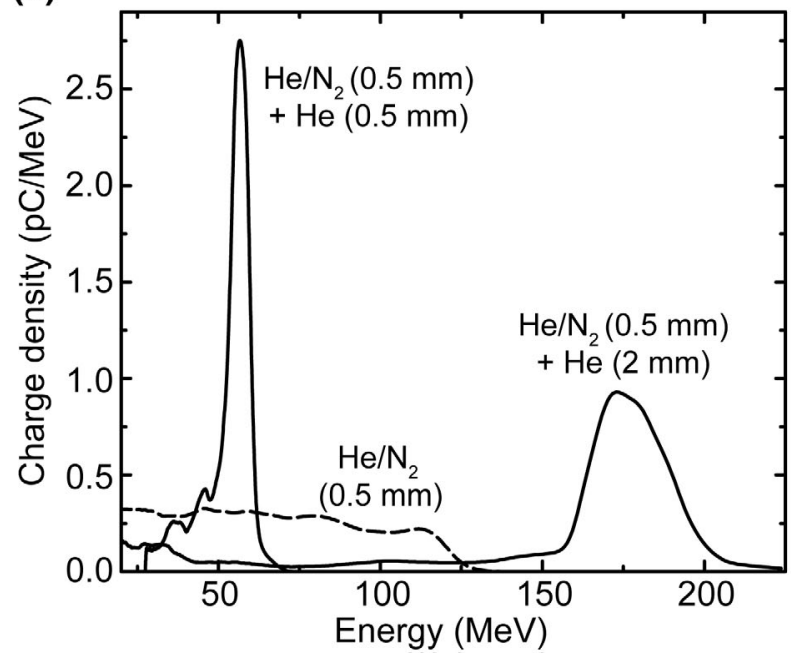

FIG. 1. (a) Gas-target profiles, measured off-line by using tomography, at a height of 2 mm above the nozzle orifices. The concentration of $\mathrm{N}_{2}$ is represented qualitatively by the colors under the curves. Roman numerals indicate different regions of the optimal composite profile (furthest back in the background), as discussed in the main text. The inset shows the orientation of the laser pulse propagation with respect to the gas target. (b) Examples of $e$-beam spectra, corresponding to these profiles. Black dashed curve: The first nozzle alone $\left(0.5\right.$-mm length, mixed gas, $\left.n_{1 e}=3.4 \times 10^{18} \mathrm{~cm}^{-3}\right)$. Black dotted curve: The second nozzle alone (2-mm length, helium, $n_{2 e}=1.2 \times 10^{18} \mathrm{~cm}^{-3}$ ). Black solid curve: Composite profile from both nozzles. In the case of the 2-mm second nozzle, the densities are the same as above; in the case of the $0.5-\mathrm{mm}$ second nozzle, $n_{1 e}=2.0 \times 10^{18} \mathrm{~cm}^{-3}$ and $n_{2 e}=1.5 \times 10^{17} \mathrm{~cm}^{-3}$.

Supplemental Material [31]). The gas used was either pure helium or a mixture of $\mathrm{He} / \mathrm{N}_{2}(99 \% / 1 \%)$. A magnetic spectrometer was used to characterize the energy of the accelerated electrons (see Supplemental Material for details).

We studied the dependence of both electron injection and acceleration on various gas profiles, through measurements of the resulting electron beam parameters. First, we used a single nozzle to create a quasi-Gaussian gas density profile [see Fig. 1(a), black dashed curve]. When a gas mixture $\left(\mathrm{He} / \mathrm{N}_{2}=99 \% / 1 \%\right)$ at electron density $n_{1 e}=(2.0-3.2) \times$ $10^{18} \mathrm{~cm}^{-3}$ was used, we observed $e$ beams with a continuous energy spectrum and an energy cutoff at 100$150 \mathrm{MeV}$ [see Fig. 1(b), black dashed curve]. This result is typical for continuous ionization-assisted injection $[25,26,32,33]$. Further evidence supporting the key role of this injection mechanism was the fact that no highenergy electrons were observed when pure helium at the same densities was used. Also, for the densities used in this experiment, the acceleration gradient [34] is estimated to be $\sim 150 \mathrm{MeV} / \mathrm{mm}$, so the maximum electron energy gain is consistent with the acceleration gradient and the gas profile.

Next, we studied the process of wakefield acceleration with more complex composite profiles, obtained from the merged flow of both nozzles. We found that only certain optimal profiles produced quasimonoenergetic $e$ beams. An example optimal profile (for the 2-mm second nozzle) is shown in Fig. 1(a), black solid curve. This profile is comprised of three distinct regions with different gradients in density and concentration of $\mathrm{N}_{2}$ relative to $\mathrm{He}$ (represented qualitatively by the color gradient under the curve). The spectrum of the beams (with central energy at $\sim 175 \mathrm{MeV}$ and a FWHM energy spread of $\sim 30 \mathrm{MeV}$ ) generated with this profile is shown in Fig. 1(b), black solid curve. A similar profile, differing only by the shorter length of the orifice of the second nozzle $(0.5 \mathrm{~mm})$, produced $e$ beams with a $\sim 70-\mathrm{MeV}$ central energy and a $\sim 15-\mathrm{MeV}$ (FWHM) energy spread [see Fig. 1(b), black solid curve]. When pure helium was used in both nozzles (for all densities shown in the paper), we did not observe a substantial amount of accelerated charge. This indicates that the operative injection mechanism was ionization-assisted injection and not self-injection or density-down-ramp injection.

The observation of a dramatically decreased electron energy spread for the optimal composite profile can be explained by the different properties of its three distinct regions [see Fig. 1(a)] and their effects on the various LWFA mechanisms. Region I $(x<-0.3 \mathrm{~mm})$ has a densitygradient up ramp composed of mixed gas. The up ramp allows the plasma wave to grow but leads to continuous bubble compression, preventing injection into the acceleration bubble (bucket) [35]. However, the density down ramp, composed of mixed gas in region II $(-0.3 \mathrm{~mm}<x<0.4 \mathrm{~mm})$, allows ionization-assisted injection. Region III $(x>0.4 \mathrm{~mm})$ contains pure helium, so ionization injection is again prevented but wakefield acceleration and electron phase-space rotation are allowed. The optimal composite profile results in narrow energy spread $e$ beams, because injection is restricted 
(a)

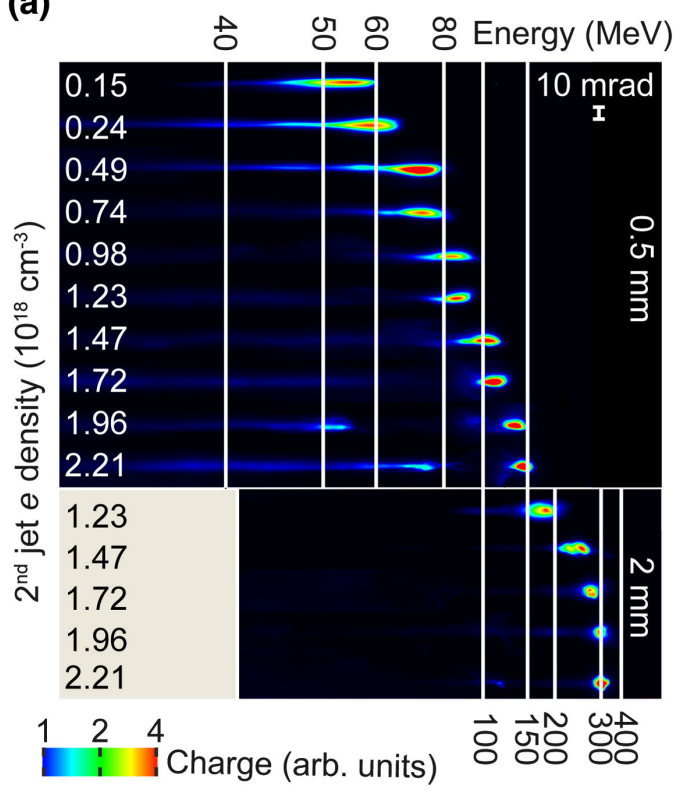

(b)

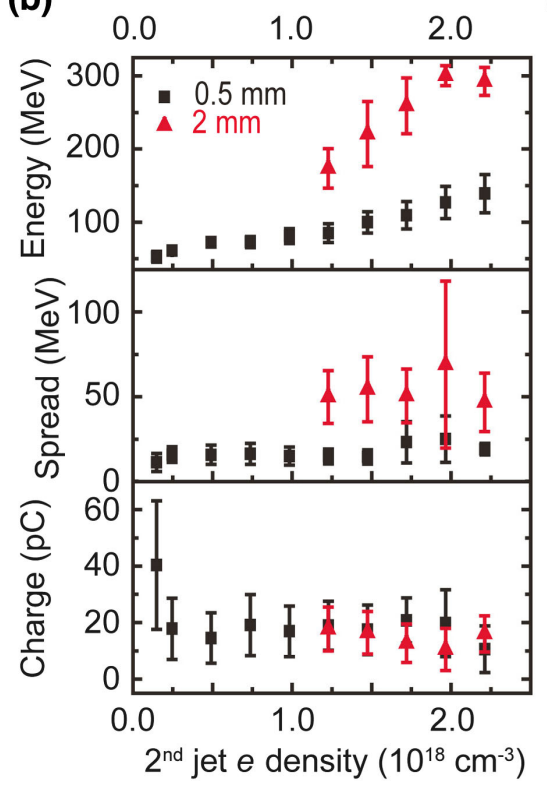

(c)

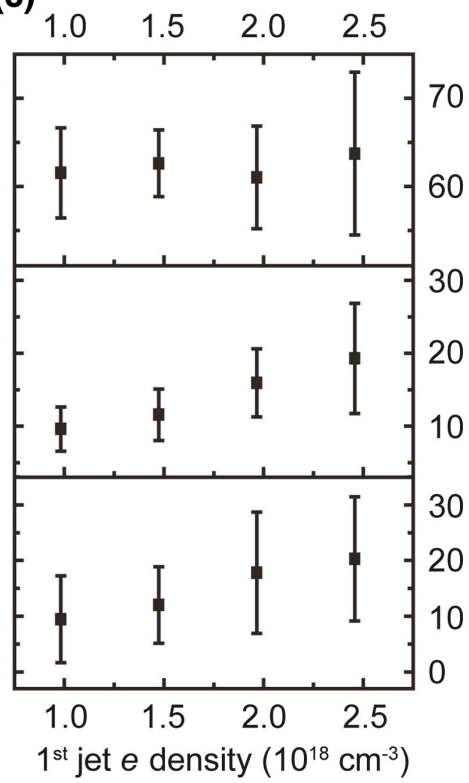

FIG. 2. (a) Typical Lanex images of the dispersed $e$ beams for different plasma densities. Top: $0.5-\mathrm{mm}$ second nozzle (the first jet $n_{1 e}=2.0 \times 10^{18} \mathrm{~cm}^{-3}$ ). Bottom: 2-mm second nozzle (the first jet $n_{1 e}=3.4 \times 10^{18} \mathrm{~cm}^{-3}$ ). The $e$-beam central energy, energy spread (FWHM), and charge as a function of electron density of (b) the second jet (the first jet $n_{1 e}=2.0 \times 10^{18}$ and $3.4 \times 10^{18} \mathrm{~cm}^{-3}$ for the $0.5-\mathrm{mm}$ and 2-mm second nozzles, respectively) and (c) the first jet $\left(0.5-\mathrm{mm}\right.$ second jet $\left.n_{2 e}=2.5 \times 10^{17} \mathrm{~cm}^{-3}\right)$. (b) and (c) show data taken from 667 experimental shots. The error bars represent the standard deviation.

exclusively to a single short region (region II). However, the quasi-Gaussian, mixed-gas profile did not result in narrow energy spread $e$ beams, because the initial up ramp, which prevents injection, was very short, and thus injection started earlier and occurred continuously.

We next demonstrated the $e$-beam central energy tunability over a large range without changing the other beam parameters, as shown in Fig. 2. We found that the optimal profile shape was qualitatively similar to the one shown in Fig. 1(a), regardless of the absolute densities of the two jets or the length of the second jet (which primarily determines the length of region III).

As can be seen from Fig. 2, the central energy increased from 50 to $140 \mathrm{MeV}$ when we tuned the density of the second gas jet ( $0.5-\mathrm{mm}$ nozzle) from $n_{2 e}=0.15 \times$ to $2.2 \times$ $10^{18} \mathrm{~cm}^{-3}$ (for fixed density of the first jet, $n_{1 e}=2.0 \times$ $10^{18} \mathrm{~cm}^{-3}$ ). The energy increased monotonically from 170 to $300 \mathrm{MeV}$ when we tuned the density of the second jet (2-mm nozzle) from $n_{2 e}=1.2 \times$ to $2.2 \times 10^{18} \mathrm{~cm}^{-3}$ (for fixed first jet density, $n_{1 e}=3.4 \times 10^{18} \mathrm{~cm}^{-3}$ ). It is reasonable that the central energy of the $e$ beams increases with the plasma density and length of the second jet, since the average accelerating field of the loaded wake $E_{\text {acc }} \propto n_{e}^{1 / 2}$ [36] and the electron energy gain is proportional to the acceleration length.

The observed saturation in the increase in central energy that was reached when the $2-\mathrm{mm}$ second nozzle was operated at $n_{2 e}=2 \times 10^{18} \mathrm{~cm}^{-3}$ [see Fig. 2(b), top panel] shows that the acceleration length matched the dephasing length in this case. The average density of this target profile $\left(n_{e}=3.5 \times 10^{18} \mathrm{~cm}^{-3}\right)$ gives an estimated dephasing length of $\sim 3 \mathrm{~mm}$, which is close to the full length of the second jet. The central energy continued to increase in the case when the $0.5-\mathrm{mm}$ second nozzle was used, since the acceleration length was much shorter in this case, and the dephasing limit was not reached. At the same time, the $e$ beam's central energy was observed to be independent of the first jet plasma density [see Fig. 2(c)], confirming that acceleration occurred primarily in region III.

The beam charge $(\sim 20 \mathrm{pC})$ remained relatively constant as the $e$-beam energy increased. The charge was independent of plasma density and the length of the second jet but depended linearly on the first jet plasma density [see Figs. 2(b) and 2(c), bottom panels]. This result confirms that injection occurred in region II and was primarily determined by the amount of nitrogen in that region. The $e$ beam's absolute energy spread shows similar behavior-it depends linearly on the first jet density and is independent of that of the second jet. This is a result of an interplay between the acceleration gradient in the injection region (region II) and its length. The energy spread (FWHM) of the $e$ beams resulting from usage of the $0.5-\mathrm{mm}$ second nozzle was $\sim 15 \mathrm{MeV}$ (or $29 \%$ and $11 \%$ for the central energies of 50 and $140 \mathrm{MeV}$, respectively) and $\sim 50 \mathrm{MeV}$ for the 2-mm one (or 29\% and $17 \%$ for the central energies of 170 and $300 \mathrm{MeV}$, respectively).

The observed trends allow one to conclude that we successfully separated injection from acceleration and can 
control the two processes independently. First, injection (and therefore charge and energy spread) could be controlled by the mixed-gas jet from the first nozzle. Our work differs from the works on down-ramp injection $[18,19,21-23]$, where only the position of injection was tuned, which did not extend to charge and energy spread tunability. Second, the acceleration length and gradient (and therefore electron energy) could be controlled by the length and density of the helium jet from the second nozzle. The measured stability and reproducibility of the $e$ beams were above $75 \%$, based on the number of shots that produced quasimonoenergetic beams with negligible tails.

To gain insight into the injection and acceleration processes, we performed simulations in $2 \mathrm{D}$ slab geometry using the OSIRIS particle-in-cell code [37]. We used a $100-\mu \mathrm{m} \times$ $200-\mu \mathrm{m}$ simulation box, with $0.015625-\mu \mathrm{m} \times 0.25-\mu \mathrm{m}$ cell size, and eight simulation particles in each cell initially. The time interval between the two steps was $0.052 \mathrm{fs}$. We utilized the moving window technique, so that a relatively small simulation box could represent a large acceleration distance. The laser beam was $0.8-\mu \mathrm{m}$ wavelength, 33 -fs pulse duration, Gaussian beam mode, with a waist of $18.69 \mu \mathrm{m}$ and a normalized vector potential of $a_{0}=2.5$, and focused at the overlap of the two gas jets. The gas-target profile was set to be the fitting result of the tomography measurement [see Fig. 1(a); $e$ densities are $3.4 \times 10^{18}$ and $1.2 \times 10^{18} \mathrm{~cm}^{-3}$ for the first and second jets, respectively], which was approximated by the summation of superGaussian and Gaussian functions. The simulation stopped at the exit of the second jet, where the gas density was low enough that neither further acceleration nor phase-space evolution was observed.

The simulations show that all accelerated electrons originated from the ionization of nitrogen. The electrons born from helium contributed to a low-energy background and were not accelerated. This correlates with our experimental observations and confirms the major role of the ionization-assisted injection. Figure 3(a) shows a quasimonoenergetic spectrum of the simulated $e$ beam, with a central energy of $\sim 200 \mathrm{MeV}$ and a FWHM energy spread of $\sim 50 \mathrm{MeV}$. This is in good agreement with the experimentally measured central energy $(170 \pm 30 \mathrm{MeV})$ and energy spread $(50 \pm 15 \mathrm{MeV})$. Figure 3(b) shows the dependence of the accelerated electrons final energy on the longitudinal position where the electrons were injected. As one can see, electrons constituting the quasimonoenergetic peak at $200 \mathrm{MeV}$ were primarily injected in the 0-0.4-mm region, which corresponds to region II of the gas-target profile. It confirms the localization of injection in this region. By comparing the energy spread immediately after injection terminated (at $\sim 0.4 \mathrm{~mm}$ ) to that at the end of the acceleration, we found that phase-space rotation decreased the absolute energy spread by a factor of 2 .

In summary, by means of experiment and simulation, we found plasma-density and atomic-composition profiles
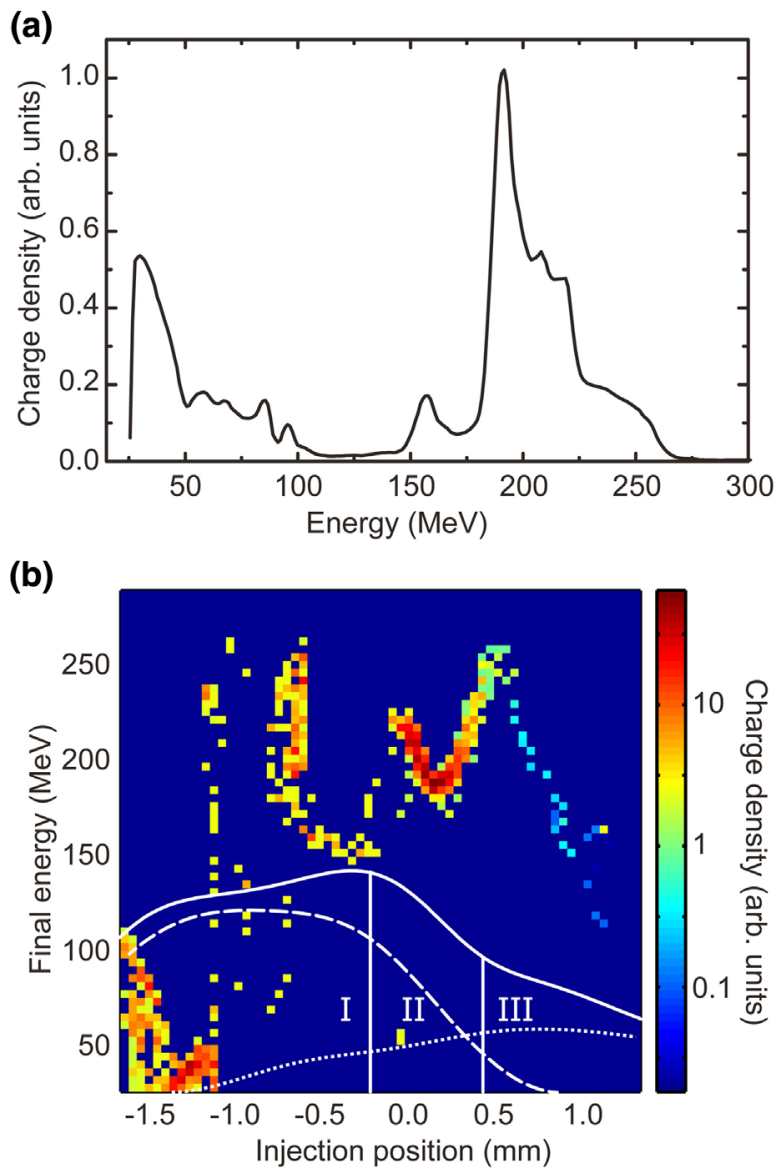

FIG. 3. Simulation results of the injected electrons at $\sim 0.9 \mathrm{~mm}$ after the phase-space evolution stops (only the electrons injected in the first bucket are considered). The electrons were originally ionized from $\mathrm{N}_{2}$ only, while those ionized from He were not injected at all. (a) Spectrum of the electron bunch. (b) The plot of electron energy vs injection position (where they were ionized). The accelerated electrons were tagged and tracked throughout the simulation to obtain this plot. The overlay shows plasma density profiles of the first jet only (dashed curve), the second jet only (dotted curve), and the combination (solid curve; Roman numerals show different regions) with the same densities shown in Fig. 1(a).

suitable for independent control of the essential LWFA mechanisms. It resulted in the generation of quasimonoenergetic $e$ beams with independently tunable energy, energy spread, and charge. This degree of control will be crucial to the application of laser wakefield accelerators in the development of the next generation of x-ray sources. The experiment and simulation provide new physical insights into the injection and acceleration processes and will result in improved performance of laser-driven electron accelerators.

We thank K. Brown, J. Mills, and C. Petersen for their contributions to the laser facility and S. Kalmykov for helpful discussions. We also acknowledge the OSIRIS Consortium, consisting of UCLA and IST (Lisbon, 
Portugal) for the use of OSIRIS and the visXD framework. Z.S. acknowledges the support by the National Basic Research Program of China (Grant No. 2013CBA01504) and the NSFC (Grants No. 11121504 and No. 11374210). This material is based on work supported by the U.S. Department of Energy (DOE), Office of Science, Basic Energy Sciences (BES), under Award No. DE-FG0205ER15663 (ultrafast x-ray science); the Air Force Office for Scientific Research, FA9550-11-1-0157 (highfield laser-electron scattering); the Department of Homeland Security Domestic Nuclear Detection Office, under competitively awarded Contract No. HSHQDC-13C-B0036 (low-dose x-ray radiography); and National Strategic Research Institute, FA4600-12-D-9000 (selective photoactivation analysis).

[1] T. Tajima and J. Dawson, Phys. Rev. Lett. 43, 267 (1979).

[2] E. Esarey, C. Schroeder, and W. Leemans, Rev. Mod. Phys. 81, 1229 (2009).

[3] K. Krushelnick and V. Malka, Laser Photonics Rev. 4, 42 (2010).

[4] V. Malka, Phys. Plasmas 19, 055501 (2012).

[5] S. M. Hooker, Nat. Photonics 7, 775 (2013).

[6] S. Corde, K. Ta Phuoc, G. Lambert, R. Fitour, V. Malka, A. Rousse, A. Beck, and E. Lefebvre, Rev. Mod. Phys. 85, 1 (2013).

[7] S. Kneip, S. Nagel, C. Bellei, N. Bourgeois, A. Dangor, A. Gopal, R. Heathcote, S. Mangles, J. Marquès, A. Maksimchuk, P. Nilson, K. Phuoc, S. Reed, M. Tzoufras, F. Tsung, L. Willingale, W. Mori, A. Rousse, K. Krushelnick, and Z. Najmudin, Phys. Rev. Lett. 100, 105006 (2008).

[8] F. Grüner, S. Becker, U. Schramm, T. Eichner, M. Fuchs, R. Weingartner, D. Habs, J. Meyer-ter-Vehn, M. Geissler, M. Ferrario, L. Serafini, B. van der Geer, H. Backe, W. Lauth, and S. Reiche, Appl. Phys. B 86, 431 (2007).

[9] H.-P. Schlenvoigt, K. Haupt, A. Debus, F. Budde, O. Jäckel, S. Pfotenhauer, H. Schwoerer, E. Rohwer, J. G. Gallacher, E. Brunetti, R. P. Shanks, S. M. Wiggins, and D. A. Jaroszynski, Nat. Phys. 4, 130 (2008).

[10] K. Ta Phuoc, S. Corde, C. Thaury, V. Malka, A. Tafzi, J. P. Goddet, R. C. Shah, S. Sebban, A. Rousse, and K. T. Phuoc, Nat. Photonics 6, 308 (2012).

[11] S. Chen, N. D. Powers, I. Ghebregziabher, C. M. Maharjan, C. Liu, G. Golovin, S. Banerjee, J. Zhang, N. Cunningham, A. Moorti, S. Clarke, S. Pozzi, and D. P. Umstadter, Phys. Rev. Lett. 110, 155003 (2013).

[12] N. D. Powers, I. Ghebregziabher, G. Golovin, C. Liu, S. Chen, S. Banerjee, J. Zhang, and D. P. Umstadter, Nat. Photonics 8, 28 (2013).

[13] J. Faure, C. Rechatin, A. Norlin, A. Lifschitz, Y. Glinec, and V. Malka, Nature (London) 444, 737 (2006).

[14] C. Rechatin, J. Faure, A. Ben-Ismail, J. Lim, R. Fitour, A. Specka, H. Videau, A. Tafzi, F. Burgy, and V. Malka, Phys. Rev. Lett. 102, 164801 (2009).

[15] S. Fourmaux, S. Corde, K. Ta Phuoc, P. M. Leguay, S. Payeur, P. Lassonde, S. Gnedyuk, G. Lebrun, C. Fourment,
V. Malka, S. Sebban, A. Rousse, and J. C. Kieffer, New J. Phys. 13, 033017 (2011).

[16] D. Umstadter, J. Kim, and E. Dodd, Phys. Rev. Lett. 76, 2073 (1996).

[17] E. Esarey, R. Hubbard, W. Leemans, A. Ting, and P. Sprangle, Phys. Rev. Lett. 79, 2682 (1997).

[18] P. Brijesh, C. Thaury, K. T. Phuoc, S. Corde, G. Lambert, V. Malka, S. P. D. Mangles, M. Bloom, and S. Kneip, Phys. Plasmas 19, 063104 (2012).

[19] S. Fourmaux, K. Ta Phuoc, P. Lassonde, S. Corde, G. Lebrun, V. Malka, A. Rousse, and J. C. Kieffer, Appl. Phys. Lett. 101, 111106 (2012).

[20] J. Faure, C. Rechatin, O. Lundh, L. Ammoura, and V. Malka, Phys. Plasmas 17, 083107 (2010).

[21] M. Burza, A. Gonoskov, K. Svensson, F. Wojda, A. Persson, M. Hansson, G. Genoud, M. Marklund, C.-G. Wahlström, and O. Lundh, Phys. Rev. ST Accel. Beams 16, 011301 (2013).

[22] K. Schmid, A. Buck, C. Sears, J. Mikhailova, R. Tautz, D. Herrmann, M. Geissler, F. Krausz, and L. Veisz, Phys. Rev. ST Accel. Beams 13, 091301 (2010).

[23] A. Buck, J. Wenz, J. Xu, K. Khrennikov, K. Schmid, M. Heigoldt, J. M. Mikhailova, M. Geissler, B. Shen, F. Krausz, S. Karsch, and L. Veisz, Phys. Rev. Lett. 110, 185006 (2013).

[24] A. J. Gonsalves, K. Nakamura, C. Lin, D. Panasenko, S. Shiraishi, T. Sokollik, C. Benedetti, C. B. Schroeder, C. G. R. Geddes, J. van Tilborg, J. Osterhoff, E. Esarey, C. Toth, and W. P. Leemans, Nat. Phys. 7, 862 (2011).

[25] J. Liu, C. Xia, W. Wang, H. Lu, C. Wang, A. Deng, W. Li, H. Zhang, X. Liang, Y. Leng, X. Lu, C. Wang, J. Wang, K. Nakajima, R. Li, and Z. Xu, Phys. Rev. Lett. 107, 035001 (2011).

[26] B. Pollock, C. Clayton, J. Ralph, F. Albert, A. Davidson, L. Divol, C. Filip, S. Glenzer, K. Herpoldt, W. Lu, K. Marsh, J. Meinecke, W. Mori, A. Pak, T. Rensink, J. Ross, J. Shaw, G. Tynan, C. Joshi, and D. Froula, Phys. Rev. Lett. 107, 045001 (2011).

[27] H. T. Kim, K. H. Pae, H. J. Cha, I. J. Kim, T. J. Yu, J. H. Sung, S. K. Lee, T. M. Jeong, and J. Lee, Phys. Rev. Lett. 111, 165002 (2013).

[28] W. Wang, W. Li, J. Liu, C. Wang, Q. Chen, Z. Zhang, R. Qi, Y. Leng, X. Liang, Y. Liu, X. Lu, R. Li, and Z. Xu, Appl. Phys. Lett. 103, 243501 (2013).

[29] C. Liu, S. Banerjee, J. Zhang, S. Chen, K. Brown, J. Mills, N. Powers, B. Zhao, G. Golovin, I. Ghebregziabher, and D. Umstadter, in Proceedings of SPIE, Solid State Lasers XXII: Technology and Devices, edited by W. A. Clarkson and R. Shori (SPIE, San Francisco, CA, 2013), Vol. 8599, pp. 859919-859919-7.

[30] C. Liu, J. Zhang, S. Chen, G. Golovin, S. Banerjee, B. Zhao, N. Powers, I. Ghebregziabher, and D. Umstadter, Opt. Lett. 39, 80 (2014).

[31] See Supplemental Material at http://link.aps.org/ supplemental/10.1103/PhysRevSTAB.18.011301 for the details on gas-jet density profile control and measurement, and electron magnetic spectrometer.

[32] C. McGuffey, A. G. R. Thomas, W. Schumaker, T. Matsuoka, V. Chvykov, F. J. Dollar, G. Kalintchenko, V. Yanovsky, A. Maksimchuk, K. Krushelnick, V. Y. 
Bychenkov, I. V. Glazyrin, and A. V. Karpeev, Phys. Rev. Lett. 104, 025004 (2010).

[33] A. Pak, K. A. Marsh, S. F. Martins, W. Lu, W. B. Mori, and C. Joshi, Phys. Rev. Lett. 104, 025003 (2010).

[34] M. Chen, E. Esarey, C. B. Schroeder, C. G. R. Geddes, and W. P. Leemans, Phys. Plasmas 19, 033101 (2012).

[35] M. Zeng, N. A. M. Hafz, K. Nakajima, L.-M. Chen, W. Lu, W. B. Mori, Z.-M. Sheng, and J. Zhang, J. Plasma Phys. 78, 363 (2012).
[36] W. Lu, M. Tzoufras, C. Joshi, F. Tsung, W. Mori, J. Vieira, R. Fonseca, and L. Silva, Phys. Rev. ST Accel. Beams 10, 061301 (2007).

[37] R. A. Fonseca, L. O. Silva, F. S. Tsung, V. K. Decyk, W. Lu, C. Ren, W. B. Mori, S. Deng, S. Lee, T. Katsouleas, and J. C. Adam, in Proceedings of Computational ScienceICCS 2002 SE - 36, edited by P. A. Sloot, A. Hoekstra, C. J. K. Tan, and J. Dongarra (Springer, Berlin, Germany, 2002), pp. 342-351. 